\title{
Breast Feeding versus Formula Feeding and Diarrheal Diseases in Infants and Children- A Review
}

\author{
MUH BEGUM
}

Summary:

The World Health Organization (WHO) and the American Academy of Pediatrics (AAP) emphasize the value of breastfeeding for mothers as well as children. Both recommend exclusive breastfeeding for the first six months of life. Human breast milk is the healthiest form of milk for babies. Breastfeeding promotes health and helps to prevent diseases including diarrheal diseases. It contains all nutrients including antibodies (IgA), and lactoferrin, that potentially prevent infection and diarrhea in infants and children. Studies conducted in both developed and under developed nations have found that breast feeding is associated with significantly ( upto 64\%) less diarrheal disease and the protective effect of breast feeding does not persist beyond two months after breast feeding is stopped. On the other hand, formula fed infants are found an upto

\section{Introduction:}

Breastfeeding and human milk are the normative standards for infant feeding and nutrition ${ }^{1}$. Breastfeeding results in improved infant and maternal health outcomes in both the industrialized and developing world. Breast milk is the best source of nutrition for the first 6 months of life. Breast milk contains all nutrients as well as antibodies especially Immunoglobulin A (Ig A) and protect baby from infections including diarrheal diseases. Though commercial infant formulas and cow's milk are considered nutritionally acceptable for infants, there is greatest risk of diarrheal diseases. Formulas and cow's milk as well as bottles, teats and utensil are attributable to contamination causing diarrheal diseases in infants who are not exclusively breastfed.

Address of Correspondence: Dr. Most. Umme Habiba Begum, Paediatric Department, Northern Private Medical College, Rangpur, Officer's Quarter no 103/6 (Sheuly), Rangpur Cantt, Mobile: 01191295626, 01712546647, e-mail: arafanis@yahoo.com

Received: 29 May, 2013

Accepted: 2 December, 2013
$80 \%$ increased in the risk of developing diarrhea compared to breast fed infants and there is significantly more diarrheal disease in formula fed infants. Infection may be attributable to contamination of bottles, teats, milk, and food in infants who are not exclusively breastfed. Exclusive breastfeeding for the first six months of life and there after complementary feedings while breastfeeding continues for up to two years of age or beyond, enthusiastic support and involvement from clinicians, obstetricians and pediatricians, are essential in "breastfeeding vs formula feeding" issue and to reduce incidence of diarrheal diseases in infants and children.

Keywords: World Health Organization (WHO), The American Academy of Pediatrics (AAP), sudden infant death syndrome (SIDS).

(J Banagladesh Coll Phys Surg 2014; 32: 26-30)

Composition of breast milk and its anti-infective role: Human breast milk is the healthiest form of milk for babies. It contains appropriate amounts of carbohydrate, protein, and fat, and provides digestive enzymes, minerals, vitamins, and hormones that infants require. Breast milk also contains antibodies from the mother that can help the baby resist infections. Colostrum and human milk contain an abundant amount of IgA ${ }^{2}$. It is secreted from the mammary and other exocrine glands during lactation. IgA prevents the attachment of bacteria and viruses to the gastrointestinal and other mucosal epithelium cells that would potentially cause infection and diarrhea. Human breastmilk also contains lactoferrin, being the main protein in human milk, This nourishment acts as a microbicidal agent killing bacteria and viruses ${ }^{3}$.

Current recommendation on breastfeeding:

As a global public health recommendation (as per WHO and AAP), infants should be exclusively breastfed for the first six months of life to achieve optimal growth, development and health. Thereafter, to meet their evolving nutritional needs, infants should receive safe 
and nutritionally adequate complementary foods while breastfeeding continues for up to two years of age or beyond ${ }^{4}$. Hospital practices that encourage successful breast-feeding include ante partum education and encouragement, immediate postpartum mother-infant contact with suckling, rooming-in arrangements, demand feeding, inclusion of fathers in prenatal breastfeeding education, and support from experienced women ${ }^{5}$. Though breastfeeding, including exclusive breastfeeding for the first 6 months of life, is widely advocated as "ideal" for babies and infants, less than $40 \%$ of infants below this age are currently exclusively breastfed worldwide ${ }^{6}$.

\section{Benefits of breastfeeding:}

Scientific research has found numerous benefits of breastfeeding for the infant. American Academy of Pediatrics research has shown that breast feeding provides advantages with regard to general health, growth, and development. Not breastfeeding significantly increases risk for a large number of acute and chronic diseases including gastroenteritis, lower respiratory infection, ear infections, bacteremia, bacterial meningitis, botulism, urinary tract infection, and necrotizing enterocolitis. They state that there are a number of studies that show a possible protective effect of breast milk feeding against sudden infant death syndrome, insulin-dependent diabetes mellitus, Crohn's disease, ulcerative colitis, lymphoma, allergic diseases, digestive diseases, and a possible enhancement of cognitive development ${ }^{7}$.

Breastfeeding is a cost effective way of feeding an infant, providing nourishment for a child at a small cost to the mother. Frequent and exclusive breastfeeding usually delays the return of fertility through lactational amenorrhea, though breastfeeding is an imperfect means of birth control. During breastfeeding beneficial hormones are released into the mother's body and the maternal bond can be strengthened. Breastfeeding is possible throughout pregnancy, but generally milk production will be reduced at some point ${ }^{8}$. The 2003 landmark Lancet Child Survival Series ranked the top 15 preventative child survival interventions for their effectiveness in preventing under-five mortality. Exclusive breastfeeding up to six months of age and breastfeeding up to 12 months was ranked number one, with complementary feeding starting at six months number three(Figure 1). These two interventions alone were estimated to prevent almost one-fifth of underfive mortality in developing countries ${ }^{9}$.

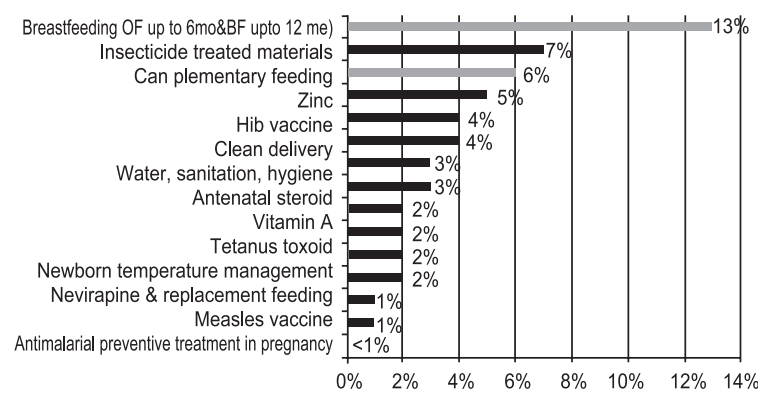

(Source: Lancet Child Survival Series 2003)

Fig.-1: Per cent of child deaths that could be prevented with $99 \%$ coverage of preventive interventions

\section{Formula feeding and health hazards:}

Besides breast milk, infant formula is the only other milk product which is under the age of one year (as opposed to cow's milk, for instance). Supplementing with solid food in addition to breast milk or formula begins during weaning, and most babies begin supplementing about the time their first teeth appear, usually around the age of six months. Although cow's milk is the basis of almost all infant formula, plain cow's milk is unsuited for infants because of its high casein content and low whey content, which may put a strain on an infant's immature kidneys, and untreated cow's milk is not recommended before the age of 12 months. The infant intestine is not properly equipped to digest non-human milk, and this may often result in diarrhea, intestinal bleeding and malnutrition ${ }^{10}$. Since the early 1970s, industrial countries have witnessed a resurgence in breastfeeding among newborns and infants to 6 months of age. This upswing in breastfeeding has been accompanied by a deferment in the average age of introduction of other foods (such as cow's milk), resulting in increased use of both breastfeeding and infant formula between the ages of 3-12 months ${ }^{11}$.

There are a number of formulas in vogue for infant feeding, such as raw milk formulas, evaporated milk formulas, commercial formulas, generic brand formulas, follow-on and toddler formulas ${ }^{12}$ etc. But use of formulas including infant formula is associated with numerous increased health risks. Studies have found infants in developed countries who consume formula are at increased risk for acute gastroenteritis, otitis 
media, severe lower respiratory tract infections, atopic dermatitis, asthma, obesity, type 1 and 2 diabetes, sudden infant death syndrome (SIDS), eczema, necrotizing enterocolitis and autism when compared to infants who are breastfed ${ }^{13}$.

\section{Breastfeeding and diarrhea:}

Breast milk is the ideal food for an infant's first six months of life. In addition to providing ideal nourishment, breastfeeding provides infants with protection from many infections, including diarrheal diseases ${ }^{14}$. Infants are at greatest risk of diarrheal disease when foods other than breast milk are given. Breastfeeding frequency should be maintained for a year or more after adding food to the infant diet to reduce the risk of diarrhea that may have serious consequences to health, nutritional status, and survival. When breastfeeding stops, infants are exposed to food-borne germs and lose the protection of breast milk's antiinfective properties such as lactoferrin, IgA, oligosaccharides. When infants do experience diarrhea, severe dehydration can occur quickly. Continued breastfeeding during diarrhea, as well as increased feeding after an episode, significantly reduces risk of dehydration, mitigates loss of weight, and promotes increased weight gain. Breastfeeding can also reduce the severity, duration, and negative nutritional consequences of diarrhea ${ }^{14}$.

\section{Discussion:}

Breast-fed children, compared with the bottle-fed ones, have a lower incidence of acute gastroenteritis due to the presence of several antiinfective factors in human milk $^{15}$. Human milk contains oligosaccharides which prevent infections related to some common pathogenic bacteria. In a study, Coppa GV, et al. has shown that fractions of oligosaccharides of human milk contains, acidic oligosaccharides, neutral high-molecular-weight oligosaccharides, and neutral low-molecular-weight oligosaccharides. The acidic fraction had an antiadhesive effect on pathogenic strains of enteropathogenic Escherichia coli serotype O119, Vibrio cholerae, and Salmonella; the neutral highmolecular-weight fraction significantly inhibited the adhesion of E. coli O119 and V. cholerae; the neutral low-molecular-weight fraction was effective toward $\mathrm{E}$. coli O119 and S. fyris. Human milk oligosaccharides inhibit the adhesion to epithelial cells not only of common pathogens like E. coli but also for V. cholerae and S. fyris. Consequently, oligosaccharides are one of the important defensive factors contained in human milk against acute diarrheal infections of breast-fed infants ${ }^{15}$.

The risk of morbidity and mortality from suboptimum breastfeeding in young children has been documented in observational studies. Robert E Black et. al. observed in a random effects meta-analysis ${ }^{16}$ that increased risk of cause-specific morbidity and mortality in relation to four patterns of breastfeeding in children younger than 6 months (exclusive-ie, nothing but breastmilk; predominant - only water or teats in addition to breastmilk; partial_-other liquids or solids in addition to breast-milk; and not breastfeeding), and two patterns (breastfeeding or not) in children aged 6-23 months. In the first 6 months of life, the relative risks were increased for each of the three patterns that were compared with the reference pattern-ie, exclusive breastfeeding, for diarrhea and pneumonia morbidity and mortality (Figure 2). The relative risks were significant for predominant breastfeeding for all-cause mortality and pneumonia incidence, and there were similar, but not significant, point estimates for diarrhea and pneumonia mortality and diarrhea incidence. Compared with exclusive breastfeeding, partial breastfeeding had moderately higher relative risks than predominant breastfeeding, and not breastfeeding had very high relative risks. In infants aged 6-23 months there was a statistically raised risk of not breastfeeding for all-cause mortality and diarrhea incidence, but there was no significant raised risk for other outcomes ${ }^{16}$.

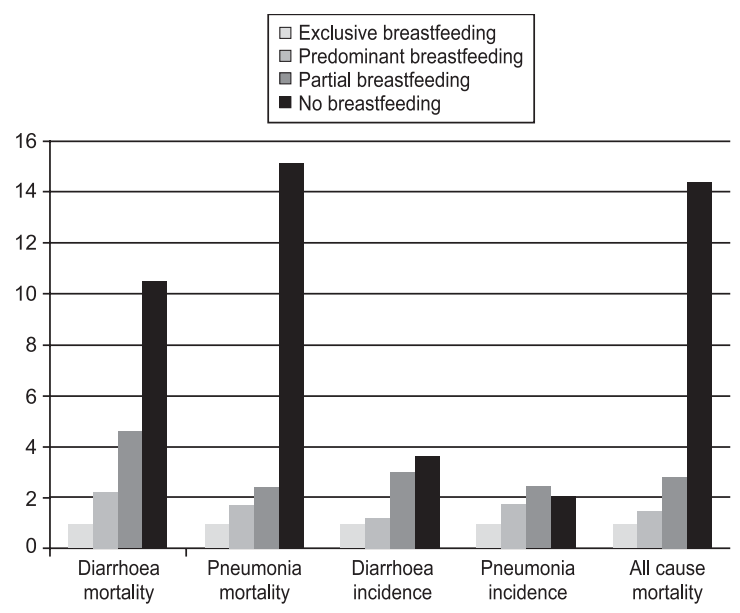

(Source: Programming Guide: Infant and Young Child Feeding; the IYCF Unit, UNICEF, June 2012)

Fig.-2: Relative risk of not breastfeeding for infections and mortality compared to exclusive breastfeeding from 0-5 months 
Case-control study of diarrheal disease among 304 infants (167 cases and 137 controls) cases presenting to 34 general practices in England showed breast feeding was associated with significantly less diarrheal disease. Associations were striking even in infants aged e" 6 months. They did not vary by social class, but were greater in those living in rented council accommodation and in more crowded households. The effect of receiving no breast milk was stronger in more deprived areas than in less deprived areas. The effect of not receiving exclusive breast milk was stronger in more deprived areas than in less deprived areas. In formula fed infants, there was significantly more diarrheal disease in those not sterilizing bottles/teats with steam or chemicals. The protective effect of breast feeding did not persist beyond two months after breast feeding had stopped. Breast feeding protects against diarrheal disease in infants in England although the degree of protection may vary across infants and wear off after breast feeding cessation. Education about the benefits of breast feeding and the risks of inadequate sterilization should be targeted at carers in deprived areas or households ${ }^{17}$. A large volume on the evidence for the many benefits of breastfeeding in industrialized countries has been compiled. It shows a $64 \%$ lowered risk for gastrointestinal tract infections ${ }^{18}$.

In the longitudinal analysis of infants in the United States conducted by Scariati, Grumer-Strawn, and Fein, researchers found an $80 \%$ increase in the risk of developing diarrhea in the formula fed children compared to breast fed infants. Investigators also found a "dose response effect that a small but steady increase in the risk of developing diarrhea as the amount of breast milk an infant received decreased"19.

An episode of diarrhea was significantly less likely to last for six or more days if an infant was breastfed for three or more months shown in a study by Baker D et al. in "Inequality in infant morbidity: causes and consequences in England in the 1990s"20.

The type of milk consumed before start of diarrhea episode was strongly associated with dehydration. Compared with infants exclusively breastfed, bottle-fed infants were at higher risk (odds ratio for cow's milk = 6.0 , for formula milk $=6.9$ ). Compared with those still breastfeeding, children who stopped in the previous two months were more likely to develop dehydrating diarrhea observed in a study by Fuchs SC et al. in "Case- control study of risk of dehydrating diarrhea in infants in vulnerable period after full weaning”21.

In the first year of life the incidence of diarrheal illness among breastfed infants was half that of formula-fed infants observed by Dewey KG et al. in "Differences in morbidity between breast-fed and formula-fed infants." study ${ }^{22}$. Children less than 12 months of age had a lower incidence of acute diarrheal disease during the months they were being breastfed than children that were fed with formula during the same period shown by Lerman,Y. et al.in "Epidemiology of acute diarrheal diseases in children in a high standard of living settlement in Israel”22. Strictly formula-fed children had an incidence of diarrhea over three times that of strictly breast-fed infants and twice that of breast-fed and supplemental fed children observed by Long KZ et al. "Proportional hazards analysis of diarrhea due to enterotoxigenic Escherichia coli and breastfeeding in a cohort of urban Mexican children”22. In the study Blake PA, et al. "Pathogen-specific risk factors and protective factors for acute diarrheal disease in urban Brazilian infants" of 500 Brazilian infants d" 12 months old with diarrhea and 500 age-matched controls, breast-feeding infants $<6$ months old (OR, 0.3) and boiling household drinking water (OR, 0.4) were protective. Breast-feeding was protective against enteropathogenic Escherichia coli infections (OR, 0.1). The addition to the breast-milk diet of even water, teas, and other nonnutritive liquids doubled or tripled the likelihood of diarrhea. Supplementation of breast-feeding with additional nutritive foods or liquids further increased significantly the risk of diarrhea observed by Popkin BM et al. in "Breast-feeding and diarrheal morbidity" study ${ }^{22}$.

Whereas breast-fed infants have less severe diarrhea when breast milk is continued rather than interrupted, infants and children fed with non-human milks tend to have more severe illness than those receiving milk-free or lactose-limited formulas or milk-cereal mixtures, observed in study by Jorge L Lembcke et al.” Effect of milk-containing diets on the severity and duration of childhood diarrhea”23.

\section{Conclusion:}

Breast milk cannot be duplicated by any artificial means. It is unique in its composition and function. Breastfeeding has been shown to reduce morbidity and mortality rates due to diarrheal diseases in infants and 
children worldwide. Different studies have consistently reported a decrease in the incidence of diarrhea in breastfed infants and children. Moreover by breast feeding, mothers are providing a natural form of nourishment, reducing long term healthcare cost, eliminating formula cost, and nurturing a bond with her child. As clinicians, recommendations should be made to soon-to-be parents regarding the importance of breast milk during routine obstetric and pediatric visits. Enthusiastic support and involvement of the pediatricians in the promotion and practice of breastfeeding is essential to reduce incidence of diarrheal diseases and thereby the achievement of optimal infant and child health, growth, and development.

\section{References:}

1. American Academy of Pediatrics: Policy Statement: Breastfeeding and the use of human milk: section on breastfeeding; Pediatrics 2012; 129(3): 827-841.

2. Islam SK, Ahmed L, Khan NI, Haque S,Begum A, Yunus AB. Immune components (IgA, IgM, IgG, immune cells) of colostrums of Bangladeshi mothers. Pediatric International; 2006; 48; 543-8.

3. Hanson LA, Korothova M, Haversen L, Mattsby-Baltzer I, Hahn-Zoric M, Silfverdal S. Breast-feeding, a complex support system for the offspring. Pediatric Int. 2002;44:34752.

4. Programming Guide: Infant and Young Child Feeding; the IYCF Unit, Nutrition Section, UNICEF New York, at: iycn@unicef.org; June 2012.

5. Waldemar A. Carlo; Nurseries and Breast-Feeding, ParentInfant Bonding; Nelson’s Text Book of Pediatrics Nineteenth edition 2011.

6. World Health Organization. World Breastfeeding Week August 1-7, 2011. Retrieved August 8, 2011.

7. Policy Stasement: Breastfeeding and the Use of Human Milk, Section on Breastfeeding; Pediatrics 2005; 115(2): 496-506;

8. "Mothers and Children Benefit from Breastfeeding". Womenshealth.gov. 27 February 2009. Archived from the original on 16 Mar 2009.

9. Jones G, Steketee R, Bhutta Z, Morris S. and the Bellagio. How many child deaths can we prevent this year? (Child Survival Series) The Lancet 2003 Vol. 362

10. Jiang, Jeter, Nelson, and Ziegler (July 2000). “Intestinal Blood Loss During Cow Milk Feeding in Older Infants". Arch Pediatr Adolesc Med. 2011; 154 (7): 673-678.
11. Ryan, Alan. "The Resurgence of Breastfeeding in the United States”.Pediatrics (American Academy of Pediatrics) 2008; 99 (4): e12.

12. From Wikipedia, the free encyclopedia; Infant formula ; last modified on 23 January 2013//http://en.wikipedia.org

13. Stanley Ip, Mei Chung, Gowri Raman, Priscilla Chew, Nombulelo Magula, Deirdre DeVine, M.Litt,Thomas Trikalinos, Joseph Lau, (April 2007). Breastfeeding and Maternal and Infant Health Outcomes in Developed Countries. Tufts-New England Medical Center Evidence-Based Practice Center. Retrieved May 22, 2008.

14. Path Factsheet: Breastfeeding and diarrhea: PATH 1455 NW Leary Way Seattle, WA USA; www.path.org/publications or email info@path.org: January 2008.

15. Coppa GV, Zampini L, Galeazzi T, Facinelli B, Ferrante L, Capretti R, Orazio G. Human milk oligosaccharides inhibit the adhesion to Caco-2 cells of diarrheal pathogens: Escherichia coli, Vibrio cholerae, and almonella fyris. Pediatr Res. 2006 Mar;59(3):377-82.

16. Robert E Black, Lindsay H Allen, Zulfiqar A Bhutta, Laura E Caulfield, Mercedes de Onis , Majid Ezzati : Maternal and child undernutrition: global and regional exposures and health consequences: The Lancet. 2008; 371: 243-260.

17. Quigley MA, Cumberland P, Cowden JM. How protective is breast feeding against diarrhoeal disease in infants in 1990s England? A case-control study. Arch Dis Child. 2006 Mar; 91(3):245-50.

18. Ip S, Chung M, Raman G, Chew P, Magula N, DeVine D, Trikalinos T, Lau J. Rockville: Breastfeeding and maternal and infant health outcomes in developed countries: US Department of Health and Human Services, Agency for Healthcare Research and Quality; 2007.

19. Scariati PD, Grummer-Strawn LM, Fein SB.A longitudinal analysis of infant morbidity and the extent of breastfeeding in the United States. Pediatrics.1997;99: 5

20. Baker D, Taylor H, Henderson J. "Inequality in infant morbidity: causes and consequences in England in the 1990s." J Epidemiol Community Health 1998;52(7):451-8

21. Fuchs SC, Victora CG, Martines J. "Case-control study of risk of dehydrating diarrhoea in infants in vulnerable period after full weaning. BMJ 1996 Aug 17;313(7054):391-4

22. Ginna Wall, MN, IBCLC; Outcome of breastfeeding vesus formula feeding: last update February 2010// gwall@u.washington.edu

23. Jorge L Lembcke, Kenneth H Brown;Effect of milk-containing diets on the severity and duration of childhood diarrhea; Acta Paediatrica; published online: 21 January 2008. 